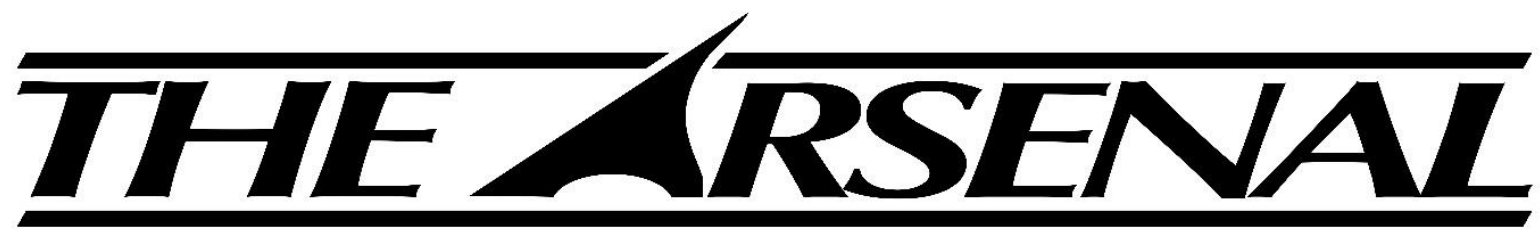

Augusta University's Undergraduate Research Journal

ISSN 2380-5064 | The Arsenal is published by the Augusta University Libraries | http://guides.augusta.edu/arsenal

Volume 3, Issue 2 (2020)

Special Edition Issue

DEGRADATION OF EGFR CONTRIBUTES TO

ANTI-CANCER EFFECTS OF HDAC INHIBITOR IN

HEAD AND NECK CANCER

Leslie Duncan, Caleb Jensen, Leilei He, Liwei Lang, and

Yong Teng

\title{
Citation
}

Duncan, L., Jensen, C., He, L., Lang, L., \& Teng, Y. (2020). Degradation of EGFR contributes to anti-cancer effects of HDAC inhibitor in head and neck cancer.

The Arsenal: The Undergraduate Research Journal of Augusta University, 3(2), http://doi.org/10.21633/issn.2380.5064/s.2020.03.02.05 


\section{Degradation of EGFR Contributes to Anti-Cancer Effects of HDAC Inhibitor in Head and Neck Cancer}

Presenter: Leslie Duncan (Poster Presentation)

Authors: Leslie Duncan ${ }^{1}$, Caleb Jensen ${ }^{1}$, Leilei $\mathrm{He}^{2}$, Liwei Lang ${ }^{2}$, and Yong Teng ${ }^{2,3}$

Faculty Sponsor(s): Yong Teng, $\mathrm{PhD}$

Department Affiliation: ${ }^{1}$ Biological Sciences, ${ }^{2}$ Oral Biology \& Diagnostic Sciences, ${ }^{3}$ Georgia Cancer Center

Funding: Augusta University Provost's Office and the Translational Research Program of the Department of Medicine

\section{ABSTRACT}

A promising arsenal of histone deacetylase (HDAC)-targeted treatment has emerged in the past decade. The abnormal targeting or retention of HDACs to DNA regulatory regions often occurs in many cancers, including head and neck squamous cell carcinoma (HNSCC); however, few cancers have been studied regarding the beneficial role of HDAC inhibition in anti-HNSCC therapy, and the underlying molecular mechanisms remain elusive. Epidermal growth factor receptor (EGFR) is commonly expressed at high levels in HNSCC (more than 90\%) and serves as a prime target for new anti-HNSCC therapy. Interestingly, Trichostatin A (TSA), one of HDAC inhibitors, not only inhibits EGFR phosphorylation, but also induces repression of EGFR total protein amount in HNSCC cells. We further show that TSA induces EGFR degradation through the ubiquitinproteasome pathway in HNSCC cells, which is associated with downregulated AKT and ERK1/2 signaling pathways. The study uncovers that EGFR is one of targets of HDACbased treatment, providing mechanistic insight into the action of HDAC inhibitors. As there is an increasing interest in using HDAC inhibitors for cancer treatment in clinics, the outcomes from the present study would be significantly beneficial for the development of new rational HDAC-targeted anticancer modalities.

Received: 01/31/2020 Accepted: 02/17/2020

Correspondence: Leslie Duncan, Augusta University, $112015^{\text {th }}$ St. Augusta, GA 30912, leduncan@augusta.edu 